\title{
Correction to: Assessing the direct and indirect effects of metalinguistic awareness to the reading comprehension skills of struggling adult readers
}

\author{
Elizabeth L. Tighe ${ }^{1}$ (D) Callie W. Little ${ }^{2}$ Meagan Caridad Arrastia-Chisholm ${ }^{3}$. \\ Christopher Schatschneider ${ }^{4}$ Emily Diehm ${ }^{5}$. Jamie M. Quinn ${ }^{4}$. \\ Ashley A. Edwards ${ }^{4}$
}

Published online: 18 December 2019

(c) Springer Nature B.V. 2019

\section{Correction to: Reading and Writing (2019) 32:787-818 https://doi.org/10.1007/s11145-018-9881-2}

In the original publication of the article, in Table 2 one of the rows was incorrectly placed. The correlation table is reported with this Correction.

The original article can be found online at https://doi.org/10.1007/s11145-018-9881-2.

\section{Elizabeth L. Tighe}

etighe@gsu.edu

1 Department of Psychology, Georgia State University, Urban Life Building, 11th Floor, 140

Decatur Street, Atlanta, GA 30303-3083, USA

2 University of New England, Armidale, Australia

3 Valdosta State University, Valdosta, USA

4 Florida State University, Florida Center for Reading Research, Tallahassee, USA

5 University of Toledo, Toledo, USA 
Table 2 Correlations among the measures

\begin{tabular}{lllllllllllllll}
\hline Measure & 1 & 2 & 3 & 4 & 5 & 6 & 7 & 8 & 9 & 10 & 11 & 12 & 13 & 14 \\
\hline 1. TOWRE-2 SWE & - & .77 & .45 & .59 & .32 & .55 & .27 & .40 & .40 & .38 & .26 & .28 & .58 & .56 \\
2. TOWRE-2 PDE & - & - & .54 & .69 & .37 & .52 & .26 & .54 & .53 & .42 & .35 & .35 & .63 & .65 \\
3. DMORPH & - & - & - & .64 & .49 & .53 & .25 & .51 & .52 & .39 & .73 & .73 & .65 & .63 \\
4. Suffix Choice & - & - & - & - & .59 & .59 & .34 & .59 & .58 & .50 & .43 & .45 & .64 & .57 \\
5. Analogy RW & - & - & - & - & - & .42 & .21 & .49 & .43 & .45 & .38 & .36 & .45 & .38 \\
6. OCRW & - & - & - & - & - & - & .34 & .38 & .30 & .33 & .40 & .39 & .54 & .53 \\
7. OCP & - & - & - & - & - & - & - & .23 & .28 & .27 & .01 & .14 & .31 & .19 \\
8. CTOPP-2 Elision & - & - & - & - & - & - & - & - & .59 & .55 & .35 & .38 & .52 & .44 \\
9. CTOPP-2 BW & - & - & - & - & - & - & - & - & - & .60 & .36 & .41 & .47 & .41 \\
10. CTOPP-2 Pho I & - & - & - & - & - & - & - & - & - & - & .27 & .29 & .40 & .38 \\
11. PPVT-4 & - & - & - & - & - & - & - & - & - & - & - & .86 & .51 & .58 \\
12. EOWPVT-4 & - & - & - & - & - & - & - & - & - & - & - & - & .49 & .54 \\
13. TABE-Reading & - & - & - & - & - & - & - & - & - & - & - & - & - & .66 \\
14. TOSREC & - & - & - & - & - & - & - & - & - & - & - & - & - & - \\
\hline
\end{tabular}

$N=200$. TOWRE-2 SWE Test of Word Reading Efficiency-Second Edition, Sight Word Efficiency, TOWRE-2 PDE Phonemic Decoding Efficiency, DMORPH Derived Form Morphology, $R W$ Real Word, $O C R W$ Orthographic Choice Real Word, OCP Orthographic Choice Pseudoword, CTOPP-2 BW Comprehensive Test of Phonological Processing-Second Edition, BW Blending Words, Pho I Phoneme Isolation, PPVT-4 Peabody Picture Vocabulary Test-Fourth Edition, EOWPVT-4 Expressive One-Word Picture Vocabulary Test-Fourth Edition, TABE Test of Adult Basic Education, TOSREC Test of Silent Reading Efficiency and Comprehension

Correlations are significant, $p<.05$

${ }^{a}$ Correlations are not significant, $p>.05$

Publisher's Note Springer Nature remains neutral with regard to jurisdictional claims in published maps and institutional affiliations. 